data to support a role for acquisition of BVAB and how this process might differ among subsets of women.

\section{S16.3 RECURRENT TV: THE POTENTIAL OF MOLECULAR TECHNIQUES TO IMPROVE CLINICAL PRACTISE}

doi:10.1136/sextrans-2013-051184.0075

M M Hobbs. University of North Carolina at Chapel Hill, Chapel Hill, NC, United States

Infection with the widespread protozoan pathogen Trichomonas vaginalis (TV) does not result in lasting immunity, and recurrent infections are common. Whether due to unrecognised, inadequately treated or repeatedly acquired TV infections, recurrent vaginitis in women, nongonococcal urethritis and chronic prostatitis in men are well-recognised and challenging clinical conditions. Despite increased recognition in the STI research community of the adverse consequences of trichomoniasis in women and men and the potential for TV infection to increase transmission of HIV and other STIs, T. vaginalis infection remains underappreciated by clinicians, public health professionals, policy makers and patients. Trichomoniasis is not a reportable STI in most countries, and TV infection is often asymptomatic, thus many infections are neither diagnosed nor treated. Symptomatic infection in women is the clinical presentation most likely to be recognised and treated, but treatment of male sexual partners of infected women is infrequent or inadequate, and testing and treatment of trichomoniasis in male patients is rare. Thus, reservoirs of infection persist.

Recent improvements in molecular diagnostics for detection of TV in women and men have the potential to improve clinical practise. Rapid antigen detection tests offer point of care testing and improved treatment options in settings where technically complex and costly nucleic acid amplification tests (NAATs) are not available, and NAATs offer highly sensitive and specific testing options for detection of TV in urogenital specimens commonly tested for other sexually transmitted pathogens including $N$. gonorrhoeae and C. trachomatis. With enhanced awareness, availability and application of these molecular tests, better detection and treatment of trichomoniasis in women and in their sexual partners can be achieved with eventual reduction of the adverse reproductive consequences associated with $T$. vaginalis infection.

\section{S16.4 LYMPHOGRANULOMA VENEREUM IN MEN WHO HAVE SEX WITH MEN. AN ONGOING EPIDEMIC SINCE 10 YEARS, BUT STILL NOT TACKLED}

doi:10.1136/sextrans-2013-051184.0076

\begin{abstract}
1,2,3H J C De Vries. 'STI outpatient clinic, Public Health Service Amsterdam, Amsterdam, The Netherlands; ' 2 Dermatology, Academic Medical Centre, University of Amsterdam, Amsterdam, The Netherlands; ${ }^{3}$ Centre for Infection and Immunity (CINIMA), Academic Medical Centre, University of Amsterdam, Amsterdam, The Netherlands
\end{abstract}

LGV is endemic in large parts of the tropics. Since 2003 anorectal LGV is also endemic among Men who have Sex with Men (MSM) throughout the industrialised world. Currently we see an increase in de incidence of LGV cases among MSM in Amsterdam. Occasional cases of heterosexual LGV are usually imported from endemic countries.

LGV is caused by Chlamydia trachomatis (Ct) biovar L. Compared to non-L biovar infections, LGV has a completely different clinical picture characterised by an invasive, lymph destructive and fibrosing inflammatory reaction. The majority of MSM with LGV are HIV co-infected (up to to $85 \%$ ), and a considerable portion is hepatitis $\mathrm{C}$ co-infected.

LGV requires extensive treatment in contrast to non-L Ct infections, thus correct biovar identification is clinically relevant. Routinely LGV is excluded in Ct positive anal, ulcer, and bubo samples. Urethral LGV is not screened routinely. The vast majority of reported LGV cases comprise anorectal infections. Infections residing at other locations than the rectum could form an undiagnosed and undertreated reservoir contributing to ongoing LGV transmission. We recently found concurrent urethral LGV infections in $2.1 \%$ of MSM with anorectal LGV. Moreover, $6.8 \%$ of the partners of anorectal LGV cases had a urethral LGV infection. This shows that urethral LGV is common, probably key in transmission, and missed in current routine LGV screening algorithms.

In European MSM the majority of LGV infections is caused by biovar L2b (Amsterdam variant). Based on clonal relatedness of prevalent LGV strains, there is evidence that the LGV epidemic among MSM prevailed already in the United States in the 1980s and was introduced into Europe by the end of the last century via the highly internationalised network of sexual contacts among MSM. A new LGV variant was unveiled and designated L2c.

\section{S16.5 MANAGEMENT OF SYPHILIS IN PREGNANCY}

doi:10.1136/sextrans-2013-051184.0077

J Wilson. Centre for Sexual Health, Leeds, UK

There are a number of clinical challenges that are specific to managing syphilis in pregnancy: Which women have the highest risk of adverse pregnancy outcome and is there anything extra we should do for them? What effect does the timing of treatment have on the pregnancy? What is the best treatment and should this be modified in the presence of HIV? What effect does the Jarisch-Herxheimer reaction have on pregnancy? What rate of adverse pregnancy outcome can be expected following successful treatment? Should all babies be treated at birth and how should the baby be monitored?

Early stage maternal infection and higher RPR increase the risk of adverse pregnancy outcome. Treatment in the third trimester is also associated with poorer outcomes. Parenteral penicillin G is the only recommended therapy for treatment of syphilis during pregnancy, and the lack of effective alternatives is why desensitisation is recommended in those who report a penicillin allergy. However, a metaanalysis concluded there is insufficient evidence to determine an optimal penicillin regimen. Adequate treatment in pregnancy significantly reduces adverse pregnancy outcomes (APOs) and congenital syphilis but APOs are still reported probably due to placental damage and effects of the fetal immune response. Some guidelines recommend treating all infants born to positive women whether or not the mother was adequately treated in pregnancy whereas others suggest this is probably not necessary. All recommend examination and serological testing of the babies every 3 months until the test/s become nonreactive.

This presentation will look at the evidence base, and the recommendations in different national guidelines, to try to provide answers to these questions.

\section{YI - American Sexually Transmitted Diseases Association - Young Investigators Symposium: research in progress: Highlights from the American STD association developmental awards programme}

\section{YI.1 CONDOM USE \& PLEASURE IN A SAMPLE OF YMSM: A CONCEPTUAL FRAMEWORK}

doi:10.1136/sextrans-2013-051184.0078

${ }^{1}$ R Arrington-Sanders, ${ }^{2} \mathrm{G}$ W Harper, ${ }^{3} \mathrm{~J} D$ Fortenberry, ${ }^{2} \mathrm{~J}$ A Bauermeister. ${ }^{1}$ Division of General Pediatrics \& Adolescent Medicine, Johns Hopkins School of Medicine, Baltimore, MD, United States, ${ }^{2}$ Health Behavior and Health Education, University of Michigan School of Public Health, Ann Arbor, MI, United States, IIndiana University School of Medicine Section of Adolescent Medicine, Indianapolis, IN, United States 